Meta

Journal des traducteurs

Translators' Journal

\title{
Les documents de normalisation
}

\section{Rosita Harvey et Michelle Rivard}

Volume 25, numéro 1, mars 1980

La documentation

URI : https://id.erudit.org/iderudit/003038ar

DOI : https://doi.org/10.7202/003038ar

Aller au sommaire du numéro

Éditeur(s)

Les Presses de l'Université de Montréal

ISSN

0026-0452 (imprimé)

1492-1421 (numérique)

Découvrir la revue

Citer cet article

Harvey, R. \& Rivard, M. (1980). Les documents de normalisation. Meta, 25(1),

126-133. https://doi.org/10.7202/003038ar d'utilisation que vous pouvez consulter en ligne.

https://apropos.erudit.org/fr/usagers/politique-dutilisation/ 


\title{
Les documents de normalisation
}

\author{
Rosita HaRvey et Michelle Rivard \\ terminologues, \\ Office de la langue française
}

Parmi la masse imposante des documents que consultent les traducteurs, les terminologues et les rédacteurs techniques au cours de leurs recherches, les normes constituent des données de référence dont il faut tenir compte pour assurer la qualité d'un texte tant sur le plan technique que sur le plan terminologique.

Mais qu'entend-on par norme? Quelle est la procédure d'établissement des documents à caractère normatif ? Est-il possible de dégager une typologie des organismes et des documents de normalisation? Enfin, comment peut-on avoir accès à ces normes et en faire la critique ? Voilà les principales questions auxquelles nous tenterons de répondre dans le cadre de cet article.

\section{PRÉCISION TERMINOLOGIQUE : DiSTINCTION ENTRE NORMALISATION TECHNIQUE ET NORMALISATION TERMINOLOGIQUE}

Nous n'entendons pas aborder ici les problèmes théoriques soulevés, entre autres, par la question de la norme linguistique, norme étroitement liée à la norme sociale et couvrant un champ beaucoup plus vaste que la norme technique et la norme terminologique. Rappelons d'abord les définitions générales de la norme (c'est-à-dire du document appelé norme) et de la normalisation telles qu'elles ont été élaborées par l'Organisation internationale de normalisation (ISO) et reprises par l'Association française de normalisation (AFNOR) dans la norme NF X03-100.

« Norme : spécification technique ou autre document accessible au public, établi avec la coopération et le consensus, ou l'approbation générale de toutes les parties intéressées, fondé sur les résultats conjugués de la science, de la technologie et de l'expérience, visant à l'avantage optimal de la communauté dans son ensemble et approuvé par un organisme qualifié, sur le plan national, régional ou international. »

«Normalisation : activité propre à apporter des solutions d'application répétitive à des questions relevant essentiellement des sphères de la science, de la technique et de l'économie et visant à l'obtention du degré optimal d'ordre dans un contexte donné. Elle se manifeste généralement par l'élaboration, la publication et la mise en application de normes. » D'autres organismes ont 
défini en d'autres termes « norme * et «normalisation», mais tous s'accordent sur les principes généraux en élargissant ou en restreignant toutefois le cadre des activités de normalisation.

Il faut par ailleurs faire la distinction entre les normes techniques qui portent sur des produits, des procédés de fabrication, des méthodes d'analyses, etc. et les normes de vocabulaire qui ont pour objet la désignation des notions. D'emblée, on constate le caractère indissociable de la normalisation technique et de la normalisation terminologique puisqu'en normalisant des «choses \$ on normalise des « mots $»$, c'est-à-dire les termes qui désignent ces réalités. Ainsi, les normes de désignation s'articulent-elles en un micro-système de la langue, apte à décrire le vocabulaire de différents domaines techniques. Par ailleurs, les techniques et les vocabulaires ont des systèmes inévitablement différents d'un pays à l'autre et c'est de l'observation de ces différences que peut naître la comparaison et, éventuellement, l'unification.

Signalons enfin que si les organismes de normalisation technique s'intéressent aussi à des questions de vocabulaire, il existe des organisations dont l'intérêt premier est la normalisation terminologique.

\section{PROCESSUS DE NORMALISATION : ÉTAPES D'ÉLABORATION D'UNE NORME TECH-} NIQUR ET D'UNE NORME DE VOCABULAIRE

Le processus de normalisation diffère selon le type d'organisme qui l'effectue, selon le pays, selon qu'il s'agit d'une norme technique ou d'une norme à caractère linguistique. \& Les traditions, le niveau de développement industriel, le système politique et bien d'autres facteurs déterminent l'orientation des travaux de normalisation nationale, et expliquent les nettes différences qui existent d'un pays à l'autre ${ }^{1}$. Malgré ces différences dans le cheminement de la normalisation et dans la structure des organismes, il serait sans doute possible de dégager un schéma-type décrivant les principales étapes d'élaboration d'une norme, qu'elle soit technique ou terminologique. Nous décrirons, à titre d'exemple, la procédure en usage à l'AFNOR pour l'établissement des normes, en conformité avec les règles de l'ISO ${ }^{2}$.

\section{$1^{\text {re }}$ étape - Travaux techniques préparatoires}

Un rapporteur et un organisme qualifié élaborent une étude, après que le commissaire à la normalisation a reconnu valable une demande qui sera inscrite au programme des travaux de normalisation. Dans le cas d'une norme terminologique, le constat de l'usage et l'énoncé du problème (cas litigieux) seront les premières données importantes.

\section{$2^{\text {e }}$ étape - Préparation du projet de norme}

Une commission formée de représentants de différents milieux intéressés examine l'étude préparatoire. On procède à des études techniques plus poussées :

1. ISO Comités membres, ISO, Genève, 1976, p. 1.

2. Les remarques se rapportant à la normalisation terminologique correspondent surtout aux travaux effectués par des organismes comme l'Office de la langue française au
Québec. 
recherches en laboratoire, essais, comparaisons avec les normes nationales et étrangères existantes, contacts avec les organismes internationaux de normalisation, etc. Puis il y a présentation d'un projet de norme approuvé provisoirement par la Commission et par l'AFNOR. Quand il s'agit d'une norme de vocabulaire, la recherche donne lieu à la rédaction d'un dossier terminologique complet.

\section{$3^{\mathrm{e}}$ étape - Enquête publique et mise au point du texte final}

L'organisme de normalisation diffuse un projet de norme auprès des principaux représentants du domaine. La Commission de normalisation examine les résultats de l'enquête et entend les auteurs qui le désirent, avant la rédaction du texte final.

Le projet de norme terminologique peut être soumis ou non à l'enquête publique. Cette enquête peut se faire auprès d'un comité d'experts ou de la Commission elle-même, ou des deux à la fois.

\section{$4^{\mathrm{e}}$ étape - Homologation}

On prépare ensuite un rapport de présentation à l'homologation avec la mention des observations non retenues. Le commissaire à la normalisation vérifie si les départements ministériels n'ont pas d'objections et le ministre compétent signe l'arrêté d'homologation qui sera publié au Journal officiel du gouvernement français. Dans certains cas, des procédures simplifiées peuvent être admises.

Toutes les normes ne franchissent pas l'étape homologation et d'ailleurs, par définition, une norme n'est pas obligatoire. Certaines normes AFNOR sont dites expérimentales, de documentation ou enregistrées. Une norme homologuée n'est obligatoire que pour les administrations publiques. Seules les normes obligatoires le sont pour tous et, dans ce cas, elles constituent des règlements.

En ce qui concerne la norme terminologique, l'intervention juridique de l'État signifie que la publication, par exemple à la Gazette officielle du Québec, rend obligatoire, dans certains textes, l'utilisation des termes normalisés.

Comme on l'a déjà mentionné, la diversité des démarches de normalisation est liée aux problèmes spécifiques de chaque pays, ce qui n'exclut pas la possibilité d'une coordination à l'échelle internationale grâce à une méthodologie de base identique.

\section{ORGANISMES DE NORMALISATION ET DOCUMENTS NORMALISÉS}

Traditionnellement, on distingue quatre niveaux de normalisation : 1) la normalisation internationale résultant de la collaboration entre plusieurs pays; 2) la normalisation régionale émanant d'un petit nombre de nations indépendantes ; 3) la normalisation nationale relevant d'un organisme reconnu comme l'autorité compétente pour établir les normes d'un pays; 4) la normalisation d'entreprise produite par les différents services d'une entreprise indépendante.

\subsection{Organismes internationaux}

L'organisation internationale de normalisation (ISO) a été créée en 1947 et elle a pour objet «de favoriser le développement de la normalisation dans 
le monde, en vue de faciliter entre les nations les échanges de marchandises et les prestations de services et de réaliser une entente dans les domaines intellectuel, scientifique, technique et économique ${ }^{3} \gg$. En 1978, l'ISO comptait 68 comités membres et regroupait les travaux de 80 pays. Elle avait publié près de 3750 normes en français, anglais et russe, la plupart dans des domaines techniques, dont 150 normes de vocabulaire.

Cependant, il existe nombre d'organismes internationaux qui exercent aussi des activités à caractère normatif et qui même quelquefois, c'est le cas de la CEI (Commission électrotechnique internationale), ont la responsabilité des normes internationales dans leurs domaines respectifs. Par exemple, l'Organisation de l'aviation civile internationale (OACI) publie des normes touchant la construction d'aéroports, la navigation aérienne, etc.

L'ISO a publié un Répertoire des organismes internationaux à activitês normatives (ISO, Genève, 1977, 68 p.). De plus, on trouvera en bibliographie quellques-uns de ces ouvrages de type répertoire qui donnent la liste d'organisations internationales ou nationales s'intéressant à la normalisation.

\subsection{Organismes régionaux}

Rappelons que ces organismes regroupent un certain nombre de pays souverains. Ainsi, le Comité européen de normalisation (CEN), fondé en 1961, regroupe les représentants des organismes nationaux de normalisation des pays de la Communauté économique européenne (CEE) et de l'Association européenne du libre échange (AELE). Le statut des normes émanant du CEN est important puisque chaque pays membre s'engage à les prendre comme normes nationales après leur adoption.

A titre d'exemples supplémentaires, signalons l'existence du Comité consultatif des normes asiatiques (ASAC) et de l'Organisation arabe de normalisation et de métrologie (ASMO).

\subsection{Organismes nationaux et associations professionnelles}

En 1901 fut créé, en Angleterre, le premier des organismes nationaux de normalisation : le British Standards Institution (BSI). Les années dix, puis les années vingt, virent apparaître en Europe plusieurs de ces organismes nationaux. En 1926, l'ISA (International Standards Association) fit son apparition pour devenir, en 1947, l'Organisation internationale de normalisation (ISO) que nous connaissons actuellement et qui réunit 80 pays formant ses comités membres.

L'Association française de normalisation (AFNOR) et le Conseil canadien des normes dont font partie, entre autres, l'Association canadienne de normalisation (ACNOR) et le Bureau de normalisation du Québec (BNQ) sont des exemples d'organismes nationaux.

3. ISO Constitution, ISO, Genève, 1976, p. 1. 
L'AFNOR, fondée en 1926, est une association privée, reconnue d'utilité publique, qui a succédé à la Commission permanente de standardisation (CPS). Elle « établit et conduit vers leur statut officiel tous les documents nationaux de normalisation, qu'ils soient préparés par ses propres commissions ou par l'un des 34 bureaux de normalisation officiellement agréés en tant qu'agents techniques d'élaboration des normes françaises et rattachés à des groupements professionnels, instituts de recherche ou centres techniques ${ }^{4} \gg$. On voit ici l'importance des liens entre les associations professionnelles et l'AFNOR, organisme qui en plus d'assurer la publication de près de 10000 normes techniques et de 500 normes de vocabulaire, joue un rôle très actif au sein de l'ISO.

Le Conseil canadien des normes (CCN), créé en vertu d'une loi du Parlement adoptée au mois d'octobre 1970, est financé par des crédits parlementaires. "... il est une corporation statutaire qui ne dépend du gouvernement ni pour sa politique ni pour ses opérations ${ }^{5}$. $\gg$ Le Conseil est un organisme de coordination dont l'objectif est de favoriser la croissance du commerce intérieur et extérieur. Ses membres sont choisis à la fois dans les milieux gouvernementaux et au sein de l'industrie et des groupements de consommateurs qui représentent nombre d'intérêts et de disciplines. Le CCN travaille en étroite collaboration avec les organismes-membres qui rédigent les normes et qui effectuent les essais. De plus, il a établi des critères auxquels les normes doivent satisfaire si elles veulent être reconnues comme normes nationales du Canada. \& Sur le plan international le Canada a un statut de participant auprès de 91 comités, et celui d'observateur auprès des 30 comités de l'Organisation internationale de normalisation; il participe également au travail de 68 des 70 comités de la Commission électrotechnique internationale ${ }^{6} . \gg$ En 1976 , lee CCN avait approuvé l'accréditation de 5 organismes rédacteurs de normes : l'Association canadienne du gaz (ACG (privé, Ont.), loffice des normes du gouvernement canadien (ONGC) (gouvernemental, Ont.), l'Association canadienne de normalisation (ACNOR) (privé, Ont.), les Underwriters' Laboratories of Canada (ULC) (privé, Ont.) et le Bureau de normalisation du Québec (BNQ) (gouvernemental).

Le Bureau de normalisation du Québec est un organisme gouvernemental rattaché au ministère de l'Industrie, du Commerce et du Tourisme. Créé en 1961, il a d'abord eu pour but de «préparer des spécifications d'achat destinées à faciliter le travail du Service général des achats et des services d'achat propres à chacun des ministères et organismes gouvernementaux ${ }^{7}$ ». Le BNQ a, depuis sa fondation, élargi son mandat et s'occupe maintenant de normalisation telle qu'on l'entend aujourd'hui. Il fait partie du Conseil canadien des normes et est le seul organisme du CCN qui soit situé en dehors de l'Ontario. Le rôle du BNQ consiste donc, d'une part, à élaborer des normes, d'autre part à mettre sur pied des systèmes de certification, c'est-à-dire de vérification périodique de la conformité des produits aux normes.

4. Catalogue des normes AFNOR, 1979, Paris, 1979, p. 2.

5. Qu'est-ce que la normalisation?, Conseil canadien des normes, sept. 1976, p. 11.

6. Qu'est-ce que la normalisation?, p. 13

7. Le $B N Q$ \& c'est quoi »?, par Jacques Verreault, (dépliant), oct.-nov. 1976. 


\subsection{Entreprises privées}

Les normes d'entreprises répondent aux besoins spécifiques d'une entreprise et ont, par conséquent, une portée plus restreinte que celles des autres types d'organismes déjà signalés. Certains services de l'entreprise sont particulièrement touchés par ces normes, tels les services de la fabrication, du contrôle de la qualité ou encore de la sécurité. Ces normes visent, entre autres, à accroître la rentabilité de l'entreprise et font l'objet de révisions périodiques. Habituellement limitées au fonctionnement interne de l'entreprise, il arrive quelquefois qu'elles soient le résultat d'ententes entre plusieurs entreprises cuvrant dans la même sphère d'activités ; c'est le cas, en France, des Sociétés Peugeot, Citroën, Renault et Simca qui se sont réunies, pour certains aspects de leurs activités, au sein de la Commission de normalisation de l'outillage et de la machineoutil (CNOMO).

$\mathrm{Au}$ Québec, les entreprises ne sont pas généralement regroupées de la même façon, mais elles participent aux travaux des organismes canadiens ou québécois de normalisation en faisant partie, par exemple, des comités d'élaboration des normes de ces organismes. Par ailleurs, dans le cadre des travaux de terminologie entrepris au Québec, elles forment parfois des comités interentreprises qui travaillent à l'obtention d'un consensus sur leur vocabulaire commun (cf. 4. La Commission de terminologie de l'Office de la langue française).

\section{ORGANISMES DE NORMALISATION TERMINOLOGIQUE}

Comme on l'a vu jusqu'à maintenant, un certain nombre d'organismes de normalisation technique s'intéressent aussi à la normalisation terminologique. Il existe, par ailleurs, diverses associations qui ont pour objet premier la normalisation terminologique. Les travaux de ces organismes résultent toujours d'un consensus de spécialistes d'une discipline donnée, quoique les processus de normalisation varient d'un organisme à l'autre.

Ainsi, le Conseil international de la langue française (CILF), fondé en 1967, rassemble les représentants de 24 pays du monde francophone. Une partie de ses travaux porte sur l'étude des vocabulaires scientifiques et techniques et les résultats des recherches des comités d'experts font l'objet de publications diverses ${ }^{8}$. Le CILF s'intéresse aussi à différents problèmes communs aux pays francophones.

À l'échelle nationale, citons les travaux des commissions de terminologie du gouvernement français. En effet, depuis 1972, le Haut comité de la langue française coordonne les recherches terminologiques entreprises au sein de différents ministères afin de combler les lacunes du vocabulaire français soit pour désigner une réalité nouvelle, soit pour remplacer des emprunts non motivés à des langues étrangères. Les commissions sont composées de représentants de l'Administration, de l'entreprise et d'institutions spécialisées. Jusqu'à maintenant, dix arrêtés ont été publiés touchant des domaines comme l'audiovisuel, les tech8. . Dictionnaire de l'Agriculture, le Vocabulaire de la radiodiffusion, le Dictionnaire fores-
tier multilingue. 
niques spatiales, l'informatique, etc. Au total 850 termes ont été traités. On a également prévu la création d'une commission générale de terminologie qui aura pour tâche principale de satisfaire aux besoins ponctuels qui ne pourront être pris en charge par les commissions spécialisées dans différents domaines.

Enfin, signalons pour la France les travaux du Comité d'étude des termes techniques français (CETTF) ainsi que ceux du Comité d'étude des termes médicaux français (CETMF) qui ont, tous les deux, publié un certain nombre de documents et articles.

$\mathrm{Au}$ Québec, la Commission de terminologie de l'Office de la langue française, créée en 1978, a un double rôle. D'une part, elle effectue des travaux ponctuels afin de répondre rapidement aux demandes du public; d'une part, elle émet sur demande des avis concernant les travaux des commissions ministérielles de terminologie et des comités interentreprises. Lorsqu'ils sont entérinés par l'Office de la langue française, les avis de la Commission de terminologie sont publiés à la Gazette officielle du Québec sous forme d'avis de recommandation ou de normalisation.

En raison de la mise en application des règlements sur la francisation des entreprises, la majorité des grosses et moyennes entreprises québécoises, qu'elles œuvrent ou non au sein des comités interentreprises, se sont dotées de services de traduction et de terminologie dont les travaux, en plus de servir à l'entreprise même, sont souvent publiés dans de nombreuses revues et font même l'objet de publications plus importantes, comme des lexiques ou des vocabulaires. Plusieurs de ces représentants de l'entreprise travaillent aussi à divers comités canadiens et québécois étroitement liés aux travaux de l'ISO.

En conclusion, on peut se demander quel est le meilleur mode d'accessibilité à ces normes et comment on peut juger de leur caractère plus ou moins normatif. D'abord, signalons qu'à l'échelle internationale il existe un système informatisé donnant des renseignements sur toutes les collections des normes nationales, des normes ISO et des documents de type normatif. Il s'agit, pour la documentation, d'ISONET (le Secrétariat central de 1'ISO assure les services de secrétariat pour le compte d'ISONET), et pour la terminologie, l'INFOTERM qui est l'organisme international chargé d'assurer la coordination des informations sur les travaux de terminologie qui se font dans le monde ${ }^{9}$. Par ailleurs, au niveau national, l'AFNOR a une banque automatisée des données terminologiques se trouvant dans les normes AFNOR et ISO constituées en un fichier appelé NORMATERM, ainsi qu'une banque documentaire comprenant, entre autres, la collection des normes AFNOR. La Banque de terminologie du Québec a aussi un fichier informatif de données terminologiques comprenant des dictionnaires, des lexiques techniques et des travaux de terminologie effectués par l'Office de la langue française, par les commissions ministérielles et par les comités interentreprises. La BTQ possède aussi un fichier inventaire qui répertorie les divers ouvrages et travaux de terminologie accessibles au Québec ou en cours de recherche, au Québec et ailleurs.

9. Adresse de ces organismes : Osterreichisches Normungsinstitut, Postfach 130 Leopoldsgasse 4, A-1021 Wien, Austria. 
Comment faire la critique de ces normes ? Est-il possible de leur attribuer une cote de pondération? Nous avons répondu partiellement à ces questions en distinguant les différents niveaux de normalisation : international, national, normes d'associations professionnelles, normes d'entreprises. Cependant, il est bon d'être critique face aux normes en tenant compte, par exemple, de la date de leur publication. De plus, soulignons certaines contraintes de l'internationalisation de la forme des termes (norme ISO/R 860-1968).

«Il peut arriver que certaines définitions de l'ISO diffèrent de celles d'un dictionnaire national : il ne faut pas alors oublier que les définitions adoptées par l'ISO sont destinées à être utilisées seulement dans le domaine spécifique de la normalisation où les termes peuvent avoir leur signification particulière ${ }^{10}$. »

C'est pourquoi il ne serait pas souhaitable que dans un désir d'uniformisation terminologique internationale, on en arrive à un para-langage inutilisable.

\section{BIBLIOGRAPHIE}

Annuaire des organisations internationales, Union des associations internationales, Bruxelles, 1974, 4297 p. (15e édition), index et suppléments.

Catalogue des normes AFNOR 1979, AFNOR, Paris, 1979, 592 p.

CEN Information 1979, CEN, Bruxelles, 1979, 51 p.

Colloque canadien sur les fondements d'une méthodologie générale de la recherche et de la normalisation en terminologie et documentation, Ottawa, les 16, 17 et 18 février 1976, publié par le Secrétariat général à la normalisation et aux affaires linguistiques, mars 1976, $308 \mathrm{p}$.

Consensus, revue du Conseil canadien des normes.

Directory of United States Standardization Activities, NBS special publication 417, U.S. Department of Commerce, National Bureau of Standards, 1975, 223 p.

ISO Comités membres, ISO, Genève, $1976,66 \mathrm{p}$.

ISO Constitution, ISO Genève, 1976, $17 \mathrm{p}$.

ISO Memento 1979, ISO, Genève, 1979, 214 p.

ISO Répertoire des organismes internationaux à activités normatives, ISO, Genève, 1977, $68 \mathrm{p}$. La Normalisation dans l'entreprise, AFNOR, Paris, 1967, 295 p.

La Normalisation linguistique, Actes de colloque international de terminologie, Lac-Delage, Québec, les 16, 17, 18 et 19 oct. 1973, OLF, Éditeur officiel du Québec, sept. 1974, $253 \mathrm{p}$.

Le BNQ « c'est quoi ₹ ? (dépliant), par J. Verreault, oct.-nov. 1976.

Le Systeme de normes nationales du Canada, Conseil canadien des normes, Ottawa, Ont., 1976.

Les Cinq Prochaines Années, 1976/1980, Conseil canadien des normes, Ottawa, Ont., 1976.

NORM-INFO, Bulletin du Bureau de normalisation du Québec, ministère de l'Industrie, du Commerce et du Tourisme, Québec.

Objectifs et principes de la normalisation, édité par T.R.B. Sanders, ISO, Genève, 1972, 121 p.

Qu'est-ce que la normalisation?, Conseil canadien des normes, Ottawa, Ont., 1976.

Standards Council of Canada, Annual Report, 1978-1979.

10. Objectifs et principes de la normalisation, édité par T.R.B. Sanders, ISO, Genève, 1972, p. 19. 